Manchester has had considerable experience in the preparation of self-teaching videotapes and we have therefore prepared a special videotape as part of our work for the AUTP Working Party on the Applied Uses of Television.

Dr John Johnson has now prepared a suitable videotape in association with one of our senior registrars, Dr Susan Benbow, and this tape is available for distribution to other interested hospitals. The videotape includes sections on indications and contraindications for the treatment, obtaining consent from the patient, preparation of the patient before treatment, and details of the treatment itself. It demonstrates how to test the ECT machine itself, and shows both unilateral and bilateral electrode placement. The hazards to the patient of using an unnecessarily large stimulus current are explained, and the viewer is shown how to recognize a well modified fit.

The programme, which is 18 minutes long, can be copied on to your own blank VHS or Soni-U-Matic cassette at a charge of $£ 25.00$.

University Hospital of South Manchester

DAVID GOLDBERG

Manchester

\section{Care and maintenance of ECT electrodes}

\section{DeAR SIRS}

Following a period of unsatisfactory performance of our Ectron machine, we referred the matter to our Principal Medical Physics Technician, David Gaffrey. He found that the cable to one of the electrodes had come adrift but also that the metal surfaces of the electrodes were badly caked with dried electrolyte solution under their gauze covers. He pointed out that this would impair the efficiency of the apparatus for the production of seizures and recommended that the electrodes should be serviced every week. This seems such an obvious point and one which is probably so widely overlooked that I request these points on the care and maintenance of ECT electrodes should be brought to wider attention.

R. P. SNATTH

\section{St James's University Hospital,}

Leeds

\section{ECT electrode maintenance when using an electrolyte solution}

(1) Remove the gauze electrode covers.

(2) Remove the metal electrodes from the screw threads by rotating anti-clockwise.

(3) Examine the metal electrodes and the screws on which the electrodes are secured for white, dry electrolyte solution.

(4) Clean the metal electrodes and screw threads thoroughly, removing all deposits of dried electrolyte using a small tooth brush and soft soap solution.

(5) Dry the metal electrodes and the screw threads.

(6) When dry, re-assemble the metal electrodes to the screw threads.
(7) Replace the gauze electrode covers with new covers.

Note: If the metal electrode is badly pitted and the metal is fiaking from the electrode, replace this electrode with a new one.

\section{General notes:}

(1) Ensure that the electrode solution is kept away from the electrode handles while treatment is in progress. This solution is a conductive medium and could cause the operator to receive an electric shock. If this solution does come into contact with the electrode handles, it should be removed and the handles dried before treatment can commence.

(2) Ensure that the cables to the ECT electrodes do not become kinked or twisted as this will place a strain on the wires inside the cables and could cause the wires to break.

(3) Always have a spare set of ECT electrodes available with the ECT machine. It is nearly always the cables that fail first.

\section{Objectives in training}

\section{DeAR SiRs}

It was heartening for me, as a trainee, to read Chris Thompson's article on educational objectives in psychiatric training (Bulletin, August 1982, 6, 141). However, I would disagree with the logic of his conclusion that because the process of setting objectives for both trainees and trainers would be difficult-requiring rigorous debate between the strands of opinion within psychiatry and inevitable compromise - then it should be abandoned.

I would also suggest that it would be useful for Clinical Tutors and Consultants to examine each job within a psychiatric rotation to determine what they hope their junior staff will get from that job, other than that vague term 'experience'.

Far too often education is seen as being provided for by means of seminars and tutorials. In fact we learn most from our patients. Enquiring minds are not encouraged by slavish reading of the journals, but by a critical use of the literature and the basic sciences to answer the clinical challenges posed by our patients. This in turn can only occur in the context of close and considered supervision of a trainee's clinical experience.

King's College Hospital,

Frank Holloway

London SE5

\section{Psychiatric experts and expertise}

\section{DeAR Sirs}

Diana Brahams' article (Bulletin, July 1982, 6, 121-22) gives the misleading impression that a psychologist, $\mathrm{Mr}$ Barrie Irving, was masquerading as a psychiatrist in the witness box. As she herself states, his description as 'the doctor' was that of her learned colleague for the Crown-an 